\title{
Los superalimentos como tendencia del mercado: Un análisis de las oportunidades para las empresas exportadoras
}

\section{Superfoods as a market trend: An analysis of opportunities for exporting companies}

Richard Fabricio Encalada-Añazco

Universidad Técnica de Machala, Ecuador, Ecuador

rfencalada_est@utmachala.edu.ec

https://orcid.org/0000-0003-4750-433X

Dayana Lisbeth Peñaherrera-Vásquez

Universidad Técnica de Machala, Ecuador, Ecuador

dpenaherrera_est@utmachala.edu.ec

https://orcid.org/0000-0003-2634-9676

Mayiya Lisbeth González-Illescas

Universidad Técnica de Machala, Ecuador, Ecuador

mlgonzalez@utmachala.edu.ec

(D) http://orcid.org/0000-0002-5219-3807

Recepción: 04/11/2020 | Aceptación: 14/01/2021 | Publicación: 10/05/2021

Cómo citar (APA, séptima edición):

Encalada-Añazco, R. F., Peñaherrera-Vásquez, D. L., y González-Illescas, M. L. (2021). Los superalimentos como tendencia del mercado: Un análisis de las oportunidades para las empresas exportadoras. Innova Research Journal, 6(2), 157-179.

https://doi.org/10.33890/innova.v6.n2.2021.1627

\section{Resumen}

El dinamismo de los mercados asociado a cambios de comportamiento de los consumidores, junto al creciente interés de las personas por mantener estilos de vida saludables, ha dado lugar al auge del consumo de superalimentos. Estos cambios representan un desafío para las empresas exportadoras que deben responder adaptando sus estrategias y oferta exportable. Este trabajo tiene por objetivo analizar la tendencia de mercado enfocada en el consumo de superalimentos, mediante la revisión de artículos científicos que faciliten la identificación de oportunidades para las empresas exportadoras de alimentos. Se empleó un enfoque cualitativo, mediante la aplicación de 
los métodos teóricos inductivo-deductivo y analítico-sintético. Para el análisis se utilizaron publicaciones científicas enfocadas en la percepción y consumo de superalimentos en varios países, y estadísticas de exportación e importación. Los principales hallazgos señalan que las tendencias actuales de consumo están relacionadas con la percepción y el comportamiento del consumidor, los estudios de las variables sociodemográficas son de gran importancia para la identificación de segmentos para la apertura de nuevos mercados. Se concluye que existen mercados potenciales para la exportación de superalimentos, especialmente en países europeos que presentan una mayor concienciación por una alimentación sana y tienen un alto poder adquisitivo. Palabras claves: tendencias en el consumo de alimentos; superalimentos; oportunidades de mercado; empresas exportadoras.

\begin{abstract}
The dynamism generated between markets and consumer behavior changes, together with the growing interest of people in maintaining healthy lifestyles, has led to the boom in the consumption of superfoods. These shifts represent a great challenge for exporting companies, which must respond by adapting their strategies and export supply. The objective of this research is to analyze the market trend focused on the consumption of superfoods, by reviewing scientific articles that facilitate the identification of opportunities for food exporting companies. The current work used a qualitative approach, through the application of inductive-deductive and analytic-synthetic theoretical methods. Scientific publications focused on the perception and consumption of superfoods in various countries, and export and import statistics were considered for the analysis. The main findings indicate that current consumption trends are related to consumer perception and behavior; the study of sociodemographic variables is of great importance to identify segments for the opening of new markets. In conclusion, there are potential markets for the export of superfoods, especially in European countries that have a higher level of awareness for healthy eating and also have high purchasing power.

Keywords: trends in food consumption; superfoods; market opportunities; exporting companies.
\end{abstract}

\title{
Introducción
}

Las nuevas tendencias de consumo de alimentos reflejan variaciones en el comportamiento de las personas a nivel mundial. La atención de la población por el cuidado del medio ambiente y el estilo de vida, se manifiesta en la preocupación por la presencia de enfermedades asociadas al origen de los alimentos y a la mala alimentación. En este sentido, existe un creciente interés por mantener una dieta saludable mediante el consumo de alimentos cuyos componentes contribuyen a mejorar la nutrición, aumentar la resistencia y las defensas del organismo, además de prevenir enfermedades crónicas y desacelerar el proceso de envejecimiento (Boratyńska y Huseynov, 2017).

En consecuencia, a esta situación, el mercado mundial de alimentos con un público consumidor cada vez más exigente en cuanto a los beneficios y atributos que los productos puedan ofrecer, ha desarrollado nuevas alternativas de alimentos saludables y nutricionales producidos bajo criterios de integridad, inocuidad y sostenibilidad (Piatti et al., 2019). 
Asociado a este contexto, se observa el auge de los productos denominados funcionales y superalimentos. Estas categorías, de acuerdo a Wolfe (2009) promueven una alimentación sana, dado que contienen un elevado valor nutricional y favorecen la salud (Loyer, 2016). La reciente popularidad de la línea de superalimentos que en su mayoría son cultivados en Latinoamérica y distinguidos por su gran aporte a la salud y nutrición, los coloca como una opción para las empresas exportadoras del sector agro-alimentario que buscan satisfacer las peticiones del consumidor.

En el ámbito académico, crece el interés por el estudio de las tendencias globales de la alimentación (Ayala et al., 2020; Magrach y Sanz, 2020; Nielsen, 2016; Vos y Bellù, 2019). En las investigaciones de Küster-BoludaaI y Vidal-Capilla (2017) se recogen las actitudes del consumidor respecto a la compra de productos funcionales, Buse et al. (2017) exploran modelos de negocios para superalimentos. Entre otros, los trabajos de Delicato et al. (2019), Tacer (2019), Van den Driessche (2018) y Varzakas (2018), avalan con evidencia empírica el consumo de superalimentos.

Bajo la perspectiva empresarial, la creciente demanda internacional de los superalimentos refleja el dinamismo del mercado, lo cual acentúa las complejidades de un entorno altamente competitivo. En este sentido, las empresas exportadoras del sector alimentario se enfrentan a una serie de retos que deben gestionar para defender su participación en el mercado. Al hilo de esta premisa, la presente investigación se orienta a responder a la pregunta ¿Cuáles son las oportunidades que ofrece el mercado alimentario a las empresas exportadoras en función de la nueva tendencia de consumo de superalimentos?

Para hacer frente a la interrogante establecida, se examinan algunos aspectos como el comportamiento y preferencias del consumidor, la actitud de las empresas y su capacidad de respuesta ante las actuales predisposiciones de los mercados internacionales. Por consiguiente, el propósito de este trabajo es analizar la tendencia de mercado enfocada en el consumo de superalimentos, mediante la revisión de artículos científicos que faciliten la identificación de oportunidades para las empresas exportadoras de alimentos. Para alcanzar el objetivo, la investigación se desarrolla desde una perspectiva cualitativa, que implica la utilización de los métodos teóricos inductivo-deductivo y analítico-sintético. Se adopta como fuente de información, artículos científicos y estudios previos realizados por organismos internacionales enlazados al ámbito de la alimentación.

Este trabajo se estructura en principio por una parte introductoria, seguida de la revisión literaria que abarca definiciones y la caracterización del mercado en relación a las preferencias actuales de los consumidores. Así mismo, se describe la metodología utilizada, posteriormente se detalla la presentación de los resultados y su discusión. Para finalizar, se exponen las conclusiones donde se recogen algunas contribuciones para la toma de decisiones de las empresas exportadoras, y las posibles limitaciones de la investigación.

\section{Revisión de la literatura}

\section{Tendencias en el consumo de alimentos}


La innovación continua, las nuevas tecnologías, el crecimiento progresivo de la población mundial y sus futuras proyecciones, causan inquietudes vinculadas al futuro de la seguridad alimentaria (Organización de las Naciones Unidas para la Alimentación y la Agricultura [FAO], 2020).

Los patrones de consumo varían lentamente con el tiempo, e influyen de forma decisiva en las preferencias alimentarias (Vos y Bellù, 2019). Según Nielsen (2016) las tendencias en el consumo de alimentos presentan un constante dinamismo, y pueden reflejarse como una preferencia temporal o convertirse en una fuerza permanente en el mercado. Estas tendencias se ven condicionadas por varios factores, como el nivel de ingresos, los cambios ambientales, las dietas alteradas, aumento de la población y las variaciones demográficas (Godfray y Garnett, 2014).

Para Allievi et al. (2015) los países desarrollados presentan un aumento del consumo de productos de origen animal y alta ingesta de calorías. De acuerdo a la apreciación de Godfray et al. (2018) el consumo de alimentos a nivel mundial se ha visto marcado por un desmedido aumento en la ingestión de carnes, proveniente del incremento de la población y de los ingresos individuales. Cabe destacar que la FAO (2017) estima que para el año 2050 la población mundial alcanzaría un aproximado de 9,7 mil millones de habitantes. En este sentido, una demanda excesiva de alimentos podría conducir a cambios en la forma de alimentación. Por otra parte, siguiendo a Tilman y Clark (2014) el factor ingreso también marca la tendencia de consumo, específicamente se asocia a la ingesta de productos azucarados con exceso de calorías y grasas saturadas, que son perjudiciales para la salud.

El desarrollo económico de los países es un factor influyente en los hábitos alimenticios (Marques et al., 2018), inclinan a las personas hacia el dispendio de productos como carnes, grasas, bebidas azucaradas y alimentos ricos en calorías. Asumiendo la posición de Tilman y Clark (2014), estas tendencias son contraproducentes para la salud por sus efectos adversos. Durante el periodo de cuarentena derivado de la crisis sanitaria del COVID-19, el consumo de carnes y alimentos industrializados se incrementó, exponiendo a las personas ante una deficiencia nutricional (Bracale y Vaccaro, 2020).

De acuerdo a Nielsen (2016), la población se está enfocando en el control de su peso, el desarrollo de energía mental, problemas cardiovasculares y circulatorios, fortalecimiento del sistema inmunológico y sistema muscular. Un segmento de mercado en crecimiento busca mejorar su nutrición y prevenir enfermedades (Boratyńska y Huseynov, 2017).

Sin duda, la dinámica del consumo ha influenciado en los cambios de la industria alimentaria. Así, en los últimos años se observa que un mayor número de empresas está realizando inversiones en el desarrollo de nuevos productos para adaptarse a los requerimientos de los consumidores. La innovación es una respuesta para los segmentos de mercado que buscan una dieta saludable con alimentos libres de químicos y sustancias artificiales. En este contexto, se destacan los alimentos funcionales, orgánicos y la categoría reciente de los superalimentos. En la tabla 1 se hace referencia a la descripción de las tres principales tendencias de consumo. 


\section{Tabla 1}

Tendencias de alimentos saludables

\begin{tabular}{|c|c|}
\hline Alimentos & Descripción \\
\hline Orgánicos & $\begin{array}{l}\text { La crítica del consumidor ante el uso de fertilizantes dañinos para la salud, ha generado el } \\
\text { crecimiento de esta tendencia, recalcando su contribución con el medio ambiente (Sheng } \\
\text { et al., 2009). } \\
\text { Desde la perspectiva del consumidor se prioriza la diferenciación de alimentos (Gutiérrez } \\
\text { et al., 2012). } \\
\text { Los consumidores aprecian una alternativa de productos que articulen factores } \\
\text { ambientales, sociales y económicos (Camarena-Gómez et al., 2020). } \\
\text { El perfil psicográfico de los consumidores de productos orgánicos se relaciona con los } \\
\text { valores, creencias, actitudes y estilos de vida (Salgado Beltrán, 2019). }\end{array}$ \\
\hline Funcionales & $\begin{array}{l}\text { El término funcional se asocia a los alimentos que se actúan como conductores a un buen } \\
\text { estado de salud (Ancos et al., 2016). } \\
\text { Su apogeo surge a raíz de una mayor conciencia de los consumidores por su salud (Lau et } \\
\text { al., 2013). } \\
\text { Esta categoría de alimentos integra un alto contenido de nutrientes y otros compuestos } \\
\text { añadidos que favorecen a la salud del organismo (Küster-BoludaaI y Vidal-Capilla, 2017). }\end{array}$ \\
\hline Superalimentos & $\begin{array}{l}\text { La aparición de nuevos factores dietéticos, ha impulsado el auge de lo superalimentos, } \\
\text { considerando la desconfianza del consumidor por los alimentos procesados; esta tendencia } \\
\text { sobresale especialmente por un mejor conocimiento del consumidor y su fuerte crítica ante } \\
\text { los riesgos para la salud (Graeff Hönninger y Khajehei, 2019). }\end{array}$ \\
\hline
\end{tabular}

Nota. Elaborado a partir de los autores citados.

De lo anteriormente citado, se puede evidenciar el deseo de los consumidores por el cuidado de su salud. Por consiguiente, la industria alimentaria ha formulado varias opciones de alimentos. Estas alternativas tienen la función en común, de favorecer a la salud del organismo, no obstante, poseen propiedades que los distinguen.

Se destaca una mayor educación por parte del consumidor, en sentido de que se encuentra mejor informado sobre los alimentos y sus efectos para la salud. La atracción del consumidor muestra una inclinación hacia alimentos naturales o que no incorporen sustancias artificiales.

Desde el enfoque de Karelakis et al. (2019) el surgimiento de los alimentos funcionales se debe a los cambios en la mentalidad del consumidor en función de su salud e ingesta de contenido nutricional. La apreciación de los autores coindice en una relación entre alimentos funcionales y salud, destacando sus contribuciones para el bienestar físico y mental. Se puede considerar que el consumo de este grupo de alimentos no solo está orientado a satisfacer necesidades nutricionales, sino que también se enfoca en la prevención de enfermedades causadas por una inadecuada alimentación. 
Tanto los productos funcionales como orgánicos han evidenciado un crecimiento en su demanda. No obstante, en los últimos años, la conducta de los consumidores refleja un marcado interés por invertir en una dieta balanceada compuesta por los llamados «superalimentos». En el siguiente epígrafe se aborda el análisis de la categoría de productos objeto de interés de este trabajo.

\section{De alimento funcional a superalimento}

Como señala Varzakas et al. (2018), la ciencia ha intervenido en la alimentación para desarrollar alimentos o enriquecerlos de nutrientes con la finalidad de combatir enfermedades. Los autores Ancos et al. (2016) separan estos alimentos en dos categorías, primeramente los que se consideran similares a los tradicionales pero con propiedades que mejoran la salud, y por otra parte aquellos que sufren modificaciones para aumentar sus compuestos activos que le dan la condición de alimentos funcionales. Los estudios de Ancos et al. (2016) y Xin y Seo (2019) enfatizan que parte de los alimentos funcionales no conservan su estado natural, es decir, experimentan transformaciones y/o alteraciones para incrementar sus componentes funcionales.

Si bien los estudios recientes demuestran un mayor interés en las personas por incluir novedosos alimentos en su dieta, a su vez exponen la preocupación por los alimentos funcionales que son sometidos a procesos de trasformación. Bajo esta referencia surge con fuerza la categoría de los superalimentos. Siguiendo a Magrach y Sanz (2020) la denominación de superalimentos se debe a su elevada composición, útil para mantener una alimentación equilibrada; son alimentos que preservan su estado natural o incluyen procesos mínimos de transformación.

Según lo explica Delicato et al. (2019), llevan el prefijo «súper»si generan mejores condiciones para la salud. Durante milenios han sido utilizados de forma tradicional por su abundancia de elementos que favorecen a la salud (Loyer, 2016).

Considerando que no existe soporte científico para definir esta categoría, algunos académicos expresan que el término se utiliza para hacer alusión a una herramienta de marketing, para otorgar mayor valor a los productos (Van den Driessche et al., 2018). La Unión Europea ha restringido el uso del término superalimentos en la comercialización de productos (Tichy et al., 2020), pese a esta situación, el consumo de estos alimentos sigue incrementando principalmente en países del occidente.

En base a revisión de literatura, se puede apreciar que no existe una definición precisa para el término superalimentos. A manera de una aproximación conceptual, en la tabla 2 se recogen las propuestas de diversos autores acerca de su apreciación sobre los superalimentos. 


\section{Tabla 2}

Propuestas teóricas de superalimentos

\begin{tabular}{ll}
\hline \multicolumn{1}{c}{ Autor / Año } & \multicolumn{1}{c}{ Propuesta } \\
\hline Wolfe (2009) & $\begin{array}{l}\text { Es un conjunto de alimentos naturales con alta concentración de nutrientes e } \\
\text { ingredientes que fortifican el sistema inmunológico y otorgan vitalidad al cuerpo } \\
\text { humano. }\end{array}$ \\
\hline $\begin{array}{l}\text { European Food } \\
\text { Information Council (2012) }\end{array}$ & $\begin{array}{l}\text { El Centro Europeo de Información para productos alimenticios los describe como } \\
\text { "aquellos alimentos, sobre todo verduras y frutas, que gracias a su alto valor } \\
\text { nutricional otorgan mayores beneficios para la salud". }\end{array}$ \\
\hline Loyer (2017) & $\begin{array}{l}\text { Son alimentos propios de tradiciones indígenas andinas que tienen características } \\
\text { auténticas, exóticas y proporcionan beneficios adicionales sin causar mayor daño } \\
\text { sobre el medio ambiente. }\end{array}$ \\
\hline Varzakas et al. (2018) & $\begin{array}{l}\text { Son aquellos alimentos naturales o con reducido procesamiento, que proporcionan } \\
\text { gran cantidad de fibras, vitaminas y un sin número de elementos beneficiosos para } \\
\text { mantener una salud óptima. }\end{array}$ \\
\hline Tacer Caba (2019) & $\begin{array}{l}\text { Se describen como alimentos tradicionales que poseen propiedades funcionales } \\
\text { bajo una condición mínima de procesamiento y han sido utilizados en regiones } \\
\text { remotas a beneficio de la salud. }\end{array}$ \\
\hline
\end{tabular}

Nota. Elaborado a partir de los autores citados.

Se asume que los superalimentos son una categoría distinguida por la abundancia de nutrientes, como vitaminas y antioxidantes inherentes por condición natural. Las propiedades que contienen estos alimentos los convierten en imprescindibles para prevenir enfermedades crónicas, además que son favorables para prevenir el desgaste que produce el envejecimiento.

Las nuevas tendencias de consumo han permitido que se incremente la demanda de superalimentos en países desarrollados y en regiones remotas a sus lugares de origen. En la tabla 3 se recogen algunos de los principales superalimentos junto a su descripción.

\section{Tabla 3}

Variedades de superalimentos

\begin{tabular}{cl}
\hline \multicolumn{1}{c}{ Producto } & \multicolumn{1}{c}{ Descripción } \\
\hline \multirow{2}{*}{ Quinua } & $\begin{array}{l}\text { Chenopodium quinoa, es una especie de grano originario de la región andina, que ha } \\
\text { sido parte de la alimentación de las comunidades indígenas durante generaciones, } \\
\text { posee un contenido excepcional de nutrientes, rico en proteínas y con cualidades } \\
\text { esenciales que juegan un papel importante en la salud y nutrición de las personas } \\
\text { (McDonell, 2015). }\end{array}$
\end{tabular}


Producto

\section{Descripción}

Salvia hispánica, planta cultivada exclusivamente en el Sur de América, desde la antigüedad sus semillas se consideran una fuente de energía y han sido utilizadas por su alto valor nutritivo, además que no contienen gluten y están compuestas por vitaminas, aminoácidos, antioxidantes, entre otras sustancias beneficiosas (Sosa et al., 2016).

Euterpe oleracea Mart, son frutos que se cultiva en la amazonia sudamericana, son ricos en vitaminas, minerales y contienen un efecto potencial como antioxidante. En

Bayas de acai (Acai berry) la cultura amazónica durante años ha sido utilizado por sus propiedades curativas, además tiene la capacidad de reducir el riesgo de contraer cáncer y retarda el envejecimiento (Ekiert y Dochniak, 2015).

Lepidium meyenii, originaria de Sudamérica, es un elemento con gran concentración de sustancias como calcio, sodio, hierro y magnesio, carbohidratos y proteínas, que producen un fuerte efecto contra afecciones producidas por la anemia y el cansancio crónico; proporciona energía y vitalidad al cuerpo, actuando como reductor de estrés (Loyer y Knight, 2018).

Vaccinium corymbosum L, procedente de Norteamérica y consumido como alimento saludable desde la época colonial. Por su abundante contenido de fibra, vitaminas, antocianinas y flavonoides, es reconocido como un superalimento con múltiples beneficios que permite reducir la presión arterial y ejerce en el como un componente anticancerígeno y posee cualidades terapéuticas (Loyer, 2017).

Arthrospira platensis/Arthrospira máxima, es una especie de cianobacteria que data desde hace millones de años, se desarrolla en el agua como una microalga de color azul-verde y se considera como un suplemento esencial para la nutrición por su elevado contenido de micronutrientes como carotenoides, proteínas, vitaminas, carbohidratos, fósforo y antioxidantes, entre otros pigmentos, que lo convierten en un superalimento ideal para tratamientos de salud y prevención de enfermedades (Soni et al., 2017).

Amaranthus spp., es una especie de planta cultivada desde tiempos ancestrales por la cultura Maya, en la actualidad varias partes del mundo lo producen en distintas formas, y se consume como vegetal o grano; este alimento es una fuente rica de almidón, aminoácidos y vitaminas con funciones biológicas positivas que contribuyen a mantener un buen estado nutricional (Algara et al., 2016).

Lycium barbarum, alimento precedente de Asia y Europa, su cultivo se da principalmente en China, utilizado durante un largo tiempo como producto medicinal; en varias regiones se conoce con distintos nombres. Se considera una súper fruta caracterizada por su alto contenido de flavonoides, carotenoides, ácidos orgánicos, hierro y otros componentes que generan efectos significativos contra problemas cardiovasculares, y enfermedades como la diabetes y el cáncer (Kulczyński y Gramza-Michałowska, 2016).

Nota. Elaborado a partir de los autores citados.

De acuerdo con Ekiert y Dochniak (2015), los superalimentos se caracterizan por su calidad nutricional, compuestos bioactivos, que incluyen fibra dietética, antioxidantes, ácidos esenciales, vitaminas y minerales, esto les ha permitido actuar positivamente en el tratamiento de muchas 
enfermedades, por medio de sus efectos anticancerígenos y antivirales brindan un equilibro en las funciones del organismo, es por ello que constituyen un gran atractivo para los consumidores que optan por incluir este tipo de alimentos en su menú alimenticio.

$\mathrm{Al}$ exponer esta variedad de superalimentos se destaca que la mayor parte provienen del cultivo primitivo de la región andina de América Latina. Los superalimentos han estado presentes en la dieta diaria de los pueblos indígenas como alimentos naturales básicos y esenciales para la prevención de enfermedades.

\section{Regulaciones y Certificaciones}

Los superalimentos por ser un término utilizado de forma reciente y al no contar con una definición científicamente aceptada, están expuestos a cumplir regulaciones y disposiciones legales. A pesar de ser considerados productos alimenticios, logran diferenciarse del resto de alimentos por la particularidad de sus propiedades, para lo que resulta complejo regirse a los marcos establecidos (Loyer, 2016).

Existen ciertos productores que añaden la denominación de superalimento en sus etiquetas, únicamente para captar la atención del consumidor, pero a su vez, el etiquetado del producto facilita al consumidor su elección en función de la información que se detalle sobre los alimentos (SI, 2020).

En concordancia con Bañares Vilella (2009), la Unión Europea se encarga de regular las declaraciones nutricionales y medicinales de los alimentos; por medio de disposiciones exige que se corroboren los atributos saludables del producto de manera previa al uso del término en su etiquetado.

El (EC)1924/2006, es el reglamento vigente establecido por la Comisión Europea, al cual deben regirse las empresas del sector alimentario en el caso de que requieran remarcar en su etiqueta los beneficios nutricionales y saludables que sus productos poseen (Comisión Europea, s.f.).

La Directiva de la Comisión Europea establece una restricción al manejo de información, de manera que el consumidor no sea objeto de engaño con respecto al valor nutricional de los alimentos, con la finalidad de brindarle protección contra la publicidad engañosa (Regulation (EC) $\mathrm{N}^{\circ}$ 1924/2006 of European Parlamient and of the Council, 2006). El establecimiento de esta normativa ha llevado a las empresas a realizar estudios científicos para constatar los aportes a la nutrición y salud especificados en las declaraciones de propiedades de sus productos.

Para las empresas del sector de alimentos es fundamental cumplir con estas disposiciones legales para obtener la autorización necesaria para la comercialización de sus productos en el mercado europeo e internacional. 
Si bien, con el aumento de la demanda de superalimentos la autorización es indispensable, pero además las empresas optan por obtener certificaciones. Para Buse, et al. (2017) las certificaciones brindan confianza de tal manera permiten enlazar con las exigencias de los mercados internacionales.

Existen certificaciones que respaldan la producción de alimentos, de acuerdo al protocolo bajo el que las empresas deseen aplicar. En la tabla 4, se muestran algunas de las certificaciones más utilizadas en el sector de alimentos.

\section{Tabla 4}

Certificaciones para alimentos

\begin{tabular}{ll}
\hline \multicolumn{1}{c}{ Certificación } & \multicolumn{1}{c}{ Descripción } \\
\hline USDA Organics & $\begin{array}{l}\text { Es un certificado basado en las normas de producción orgánica de Estados Unidos, } \\
\text { utilizado para alimentos y bebidas orgánicas, y garantiza que al menos el 95\% de } \\
\text { los ingredientes del producto no contienen sustancias sintéticas o artificiales } \\
\text { (Procolombia, 2018). }\end{array}$ \\
\hline $\begin{array}{l}\text { The Rainforest Alliance } \\
\text { Certified }\end{array}$ & $\begin{array}{l}\text { Comprende un sello certificado que reciben las fincas u organizaciones que } \\
\text { cumplen con estrictas normas ambientales, y garantiza al consumidor que los } \\
\text { productos han sido cultivados bajo prácticas socialmente responsables (Vera y } \\
\text { Cañón, 2018). }\end{array}$ \\
\hline Fair trade & $\begin{array}{l}\text { Fair Trade, es una marca que avala un trato justo para los productores mediante la } \\
\text { aplicación valores éticos y adecuadas condiciones comerciales y laborales, en } \\
\text { relación a la producción, precio y salarios dignos (Proecuador, 2013). }\end{array}$ \\
\hline Logos Veganos & $\begin{array}{l}\text { Son sellos reconocidos que garantizan a los consumidores que los productos } \\
\text { cumplen con los requerimientos veganos, es decir no contienen ingredientes de } \\
\text { procedencia animal y no han sido objeto de prueba con animales. Entre estos logos } \\
\text { destacan Vegan Society y Vegan Action (SI, 2020). }\end{array}$ \\
\hline $\begin{array}{l}\text { Buenas Prácticas Agrícolas certificación que comprende normas aplicadas a la producción agrícola, } \\
\text { (BPA) }\end{array}$ & $\begin{array}{l}\text { destinadas al aseguramiento de la calidad de los productos en materia de salud e } \\
\text { higiene y contribuye a la protección del medio ambiente, mejorando así la calidad } \\
\text { de vida de las personas (Rizo-Mustelier et al., 2017). }\end{array}$ \\
\hline
\end{tabular}

Nota. Elaborado a partir de los autores citados.

De la lista expuesta se puede destacar que las certificaciones han cobrado un papel estratégico como herramientas complementarias para respaldar los procesos de producción y comercialización de productos agrícolas. Específicamente, para el caso de los superalimentos las certificaciones son necesarias para cubrir las exigencias de los principales mercados de destino.

Según señala Mora-Córdova et al. (2020), las certificaciones favorecen tanto a la empresa como a los consumidores, ya que la adopción de este mecanismo acredita la observancia de los protocolos establecidos asegurando la calidad del producto. 


\section{Metodología}

Esta investigación se desarrolla desde una perspectiva cualitativa, es de carácter descriptiva. Mediante la aplicación de los métodos teóricos inductivo-deductivo, analíticosintético, se analizan las tendencias de consumo de los superalimentos para identificar e interpretar las oportunidades que el mercado alimentario ofrece a las empresas exportadoras de alimentos.

Para organizar los resultados que permitan responder a la pregunta y objetivo de investigación, en una primera parte del estudio se realizó la revisión de 10 artículos de investigación realizados por otros autores, enmarcados en los objetivos de analizar la percepción y el comportamiento del consumidor de superalimentos en distintos mercados. Los artículos científicos seleccionados cuentan como fuente de información secundaria que se obtuvieron de las bases de datos: Elsevier, Scielo, Taylor y Francis, Redalyc, Esmerald y Agecon Search. Los artículos analizados corresponden a la percepción de los consumidores ante las tendencias del mercado y la decisión de compra de productos reconocidos como superalimentos. Se destacan variables operacionales que han permitido identificar segmentos de mercado, y los resultados consideran la relación de las variables con la generación de oportunidades para las empresas exportadoras.

Para la segunda parte de los resultados, se utilizaron estadísticas de exportación e importación de varios superalimentos. Se empleó como fuente Trade Map, que es una web interactiva de estadísticas del comercio mundial, desarrollada por el Centro de Comercio Internacional UNCTAD/OMC (CCI). Como criterio para la selección de los productos se utilizaron las referencias del CBI (2019; 2020), que es el Centro para la Promoción de Importaciones de los países en desarrollo, fundada por el Netherlands Ministry of Foreign Affairs y de Wyman (2019), el cual presenta las tendencias y estadísticas de consumo de alimentos en Europa basándose en el reporte de la Feria FruitLogística celebrada en Alemania.

Al hilo de esta justificación, se fijó la búsqueda por volúmenes de exportación de los productos: quinua, chía, arándanos, jengibre, aguacate, cúrcuma, asociados a los países que han registrado las mayores ventas.

\section{Resultados y Discusión}

En coherencia con el objetivo planteado, los resultados se presentan de forma sistematizada, mediante la recopilación de trabajos previos sobre los superalimentos, a partir de los mismos se pretende brindar respuesta a la interrogante: ¿Cuáles son las oportunidades que ofrece el mercado alimentario a las empresas exportadoras en función de la nueva tendencia de consumo de superalimentos?

Para la presentación de los resultados se muestran dos apartados. Para la primera parte, en la tabla 5 se describen 10 artículos científicos en relación al comportamiento del consumidor en el mercado y la percepción sobre los superalimentos. Se extraen datos relevantes como la muestra utilizada, el país de estudio, variables empleadas y una síntesis de los principales resultados. 


\section{Tabla 5}

Percepción del consumidor de superalimentos

\begin{tabular}{|c|c|c|c|}
\hline Autor/Año & Muestra/País & Variables & Resultados \\
\hline $\begin{array}{l}\text { Menezes et al. } \\
\text { (2011) }\end{array}$ & $\begin{array}{l}155 \\
\text { Consumidores } \\
\text { (Estados } \\
\text { Unidos) }\end{array}$ & $\begin{array}{l}\text { - Intención de compra } \\
\text { - Interés por la salud }\end{array}$ & $\begin{array}{l}\text { Los consumidores encuestados reflejan carencia de } \\
\text { conocimiento sobre los beneficios del acaí. Los } \\
\text { estudios sostienen la existencia de posibles } \\
\text { segmentos de mercado: en adolescentes, adultos } \\
\text { mayores y consumidores de sexo femenino, que } \\
\text { mantienen una gran preocupación por la salud. El } \\
\text { mercado de california se interesa en dos opciones de } \\
\text { productos, en presentación de sorbete y jugos. }\end{array}$ \\
\hline
\end{tabular}

\begin{tabular}{lll}
\hline Brečić et al. & 500 entrevistas & - Precio \\
$\mathbf{( 2 0 1 7 )}$ & cara a cara & - Salud \\
& (Europa) & - Características \\
& & sensoriales
\end{tabular}

El estudio revela que para los consumidores del rango de 41-55 años, lo más relevante es que los alimentos tengan un buen sabor y propiedades nutricionales para mejorar el estado de salud. En cuanto al segmento $<30$ años, las características extrínsecas más relevantes son el precio y la calidad, elementos considerados para los consumidores en las intenciones de compra.

\begin{tabular}{llll}
\hline $\begin{array}{l}\text { Bai et al. } \\
\text { (2019) }\end{array}$ & $\begin{array}{l}360 \text { personas } \\
\text { (China, Jilin) }\end{array}$ & $\begin{array}{l}\text { - Valor percibido de } \\
\text { intención de compra } \\
\text { - Actitudes de los } \\
\text { consumidores. }\end{array}$ & $\begin{array}{l}\text { De acuerdo con los resultados, en China la demanda } \\
\text { de ginseng es sumamente elevada, ya que se } \\
\text { considera como un alimento medicinal. El valor } \\
\text { percibido por el consumidor y su intención de } \\
\text { compra son muy altos. Los factores que influyen al } \\
\end{array}$ \\
& & $\begin{array}{l}\text { momento de adquirir este alimento son el poder } \\
\text { adquisitivo de los habitantes y el precio del producto. }\end{array}$
\end{tabular}

\begin{tabular}{|c|c|c|c|}
\hline $\begin{array}{l}\text { Birch et al. } \\
(2018)\end{array}$ & $\begin{array}{l}521 \\
\text { consumidores } \\
\text { (Australia) }\end{array}$ & $\begin{array}{l}\text { - Género } \\
\text { - Edad } \\
\text { - Educación } \\
\text { - Familiaridad } \\
\text { - Factor neo fóbico } \\
\text { - Conciencia por la } \\
\text { salud } \\
\text { - Valor simbólico }\end{array}$ & $\begin{array}{l}\text { El estudio presenta resultados alentadores, } \\
\text { considerando una gran percepción por el consumo de } \\
\text { algas, principalmente en los participantes con mayor } \\
\text { nivel educativo. Así mismo, los factores como la } \\
\text { familiaridad con el producto, el valor simbólico, la } \\
\text { conciencia por la salud y el factor neo fóbico } \\
\text { presentan valores elevados que inducen a una mayor } \\
\text { probabilidad de consumir algas marinas. }\end{array}$ \\
\hline $\begin{array}{l}\text { Meyerding et } \\
\text { al. (2018) }\end{array}$ & $\begin{array}{l}503 \\
\text { consumidores } \\
\text { mayores de } 18 \\
\text { años } \\
\text { (Alemania) }\end{array}$ & $\begin{array}{l}\text { - Genero } \\
\text { - Edad } \\
\text { - Estados Federales } \\
\text { - Tamaño del hogar } \\
\text { - Educación } \\
\text { - Ingresos }\end{array}$ & $\begin{array}{l}\text { Los resultados evidencian que los productos de } \\
\text { mayor interés para el consumidor son la chía y la } \\
\text { linaza. La mayor parte de los consumidores se } \\
\text { enfocan en la idea de adquirir un producto sano y de } \\
\text { buena calidad. Además, se demuestra que existen } 4 \\
\text { segmentos de mercado, donde la decisión de compra } \\
\text { se ve influenciada por el precio, la calidad o el deseo } \\
\text { de consumir alimentos con beneficios nutricionales. } \\
\text { Otro factor que se considera en la elección de los } \\
\text { súper alimentos, como la chía, linaza, amaranto y } \\
\text { centeno, es el tiempo de duración de estos productos. }\end{array}$ \\
\hline
\end{tabular}




\begin{tabular}{|c|c|c|c|}
\hline Autor/Año & Muestra/País & Variables & Resultados \\
\hline $\begin{array}{l}\text { Delicato et al. } \\
\text { (2019) }\end{array}$ & $\begin{array}{l}205 \text { personas } \\
\text { (Bélgica) }\end{array}$ & $\begin{array}{l}\text { - Decisión de compra } \\
\text { - Percepción de los } \\
\text { consumidores. }\end{array}$ & $\begin{array}{l}\text { Los resultados de la población encuestada arrojan } \\
\text { que el consumo de superalimentos es mayor en las } \\
\text { personas con una edad promedio superior a } 56 \text { años, } \\
\text { quienes prefieren estos alimentos por su capacidad } \\
\text { nutricional, esencial para el cuerpo humano. Debido } \\
\text { a la condición de edad del segmento encuestado, la } \\
\text { decisión de compra de superalimentos es impulsada } \\
\text { por su deseo de estar saludable y no presenta } \\
\text { influencia de las redes sociales. }\end{array}$ \\
\hline
\end{tabular}

$\begin{array}{lll}\begin{array}{l}\text { Rojas-Rivas et } \\ \text { al. (2019) }\end{array} & 610 & \text { - Salud y } \\ & \text { consumidores } & \text { funcionalidad } \\ & \text { (México) } & \text {-Características } \\ & & \text { sensoriales } \\ & & \text { - Conveniencia } \\ & & \text { - Precio } \\ & & \text { - Etiquetado }\end{array}$

En base a la revisión de resultados, los aspectos sociodemográficos trascienden en la motivación de consumo del amaranto. Así mismo, las Características Sensoriales, la Conveniencia, y el Precio, son los constructos que presentan mayor relevancia en la percepción del consumidor.

Por otra parte, la apreciación en cuanto a salud y funcionalidad, conjuntamente con la disposición de compra de productos tradicionales se consideran aspectos cruciales en el consumo de este superalimento.

\begin{tabular}{lll}
\hline $\begin{array}{l}\text { Ayala-Garay } \\
\text { et al. (2020) }\end{array}$ & 135 personas & - Cantidad \\
& (México) & consumida. \\
& & - Frecuencia de \\
& & consumo. \\
& & - Cantidad que \\
& & consume. \\
& & - Precio dispuesto a \\
& & pagar.
\end{tabular}

Los resultados indican que del total de participantes solo el 35\% conoce los atributos del amaranto. La cantidad y frecuencia de consumo están determinadas por el nivel de ingresos de las personas. Por tal, se establece que el nicho de mercado a desarrollar lo comprenden las personas que tienen su trabajo o estudio, y que presentan el deseo de consumirlo en su menú cotidiano.

\begin{tabular}{lll}
\hline Palmieria y & 257 & - Actitudes de los \\
Forleo (2020) & consumidores & consumidores. \\
& (Italia) & - Percepción de los \\
& & consumidores sobre \\
& & las algas comestibles. \\
& & - Información \\
& sociodemográfica.
\end{tabular}

Los hallazgos revelan una actitud positiva en la percepción de los participantes en cuanto al consumo de algas. Un punto propicio para establecer un nicho de mercado es Italia, debido a la elevada predisposición del consumidor por la compra de algas comestibles y su familiaridad con el producto; esto indica que el consumidor italiano presenta mayor interés por adquirir nuevos alimentos.

\begin{tabular}{lll}
\hline $\begin{array}{l}\text { Sulistyawati et } \\
\text { al. (2020) }\end{array}$ & 483 & - Preferencias del \\
& encuestados & consumidor \\
& $(137$ chinos, & - Familiaridad \\
& 244 & \\
& indonesios, & \\
& 102 & \\
& holandeses $)$ &
\end{tabular} Las variables sociodemográficas demuestran que el segmento de consumidores chinos y holandeses entre edades de 18 a 40 años presentan mayor conciencia por su salud adquiriendo productos sin ingredientes añadidos convirtiéndose en un atributo de calidad de los alimentos, en cambio los indonesios demuestran más interés en características intrínsecas como: color, textura y sabor. La cultura de los países es el principal atributo que influye en las preferencias de consumo.

Nota. Elaborado a partir de los autores citados. 
De la recopilación de los principales resultados se puede extraer que la percepción del consumidor frente a la tendencia de superalimentos es positiva. De los estudios de Rojas-Rivas et al. (2019) se destaca que las variables con mayor relevancia para medir la percepción de consumo son la intención de compra, el precio del producto, y la creciente preocupación de los consumidores por la salud.

Así también, el nivel de educación y el nivel de ingresos como elementos de gran influencia al momento de realizar la compra de superalimentos. Por otra parte, se puede determinar que las variables sociodemográficas son de gran aporte para identificar segmentos y a su vez desarrollar nichos de mercado, lo que coincide ampliamente con la literatura citada por Godfray y Garnett (2014). Los consumidores están pendientes de la innovación de productos del sector alimenticio que se diferencian por tener estos atributos (Xin y Seo, 2019).

En este sentido, con el respaldo de los estudios citados, se puede identificar que existe un segmento de mercado que se encuentra claramente influenciado por la necesidad de cambiar de estilo de vida. Las oportunidades para las empresas exportadoras se identifican en el abanico de alternativas de alimentos que puedan sostener en su oferta exportable, considerando que la conformación de dicha oferta debe estar dirigida a la salud, a la prevención de enfermedades y el respeto al medio ambiente.

Para presentar la segunda parte de los resultados, a continuación, se exponen estadísticas de exportación de los productos: quinua, chía, arándanos, jengibre, aguacate y cúrcuma. La selección de los alimentos obedece al respaldo identificado en las fuentes relacionadas con las publicaciones de Wyman (2019) y CBI (2020).

En la Tabla 6 se ubican los volúmenes de exportación del periodo (2017-2019), que reflejan a los principales países exportadores a nivel mundial, respecto a los productos seleccionados.

\section{Tabla 6}

Total de exportaciones de superalimentos por país

\begin{tabular}{|c|c|c|c|c|}
\hline \multirow[b]{2}{*}{ Productos } & \multirow{2}{*}{ País } & \multicolumn{3}{|c|}{ Toneladas exportadas } \\
\hline & & 2017 & 2018 & 2019 \\
\hline \multirow{3}{*}{ Quinua } & Perú & 52.043 & 50.084 & 48.781 \\
\hline & Bolivia & 32.347 & 33.106 & 32.145 \\
\hline & Ecuador & 1.938 & 1.719 & 2.389 \\
\hline \multirow{3}{*}{ Chía } & Paraguay & 13.681 & 14.396 & 21.972 \\
\hline & Bolivia & 12.394 & 12.909 & 9.504 \\
\hline & Perú & 6.990 & 5.736 & 5.727 \\
\hline \multirow{3}{*}{ Arándanos } & Chile & 109.329 & 141.303 & 138.372 \\
\hline & Perú & 43.039 & 72.583 & 122.449 \\
\hline & México & 13.930 & 19.206 & 28.344 \\
\hline \multirow{3}{*}{ Jengibre } & China & 440.888 & 476.775 & 523.496 \\
\hline & Tailandia & 83.248 & 73.525 & 77.797 \\
\hline & Perú & 26.409 & 20.773 & 22.801 \\
\hline Aguacate & México & 897.748 & 1.091 .936 & 1.112 .136 \\
\hline
\end{tabular}




\begin{tabular}{llrrr}
\hline \multirow{2}{*}{ Productos } & \multirow{2}{*}{ País } & \multicolumn{3}{c}{ Toneladas exportadas } \\
\cline { 2 - 5 } & Perú & \multicolumn{1}{c}{$\mathbf{2 0 1 7}$} & \multicolumn{1}{c}{$\mathbf{2 0 1 8}$} & \multicolumn{1}{c}{$\mathbf{2 0 1 9}$} \\
\cline { 2 - 5 } & Chile & 193.364 & 359.428 & 312.073 \\
& Colombia & 28.487 & 144.422 & 156.973 \\
& India & 108.312 & 122.009 & 44.570 \\
\hline \multirow{3}{*}{ Cúrcuma } & Indonesia & 7.795 & 9.541 & 130.063 \\
& Perú & 1.837 & 1.510 & 7.163 \\
& & & & 1.938 \\
\hline
\end{tabular}

Nota. Elaboración propia, adaptado de International Trade Center (ITC, 2020a), Trade Map.

En consistencia con Loyer (2016), se confirma que el origen de las exportaciones de los cuatro superalimentos seleccionados, proviene de países latinoamericanos como es el caso de Perú, Bolivia, Ecuador, Chile y México.

El auge de los productos alimenticios señalados es una tendencia que representa oportunidades para los países productores. De forma complementaria, existe evidencia que señala un incremento en los programas de certificaciones de responsabilidad social, como una estrategia para respaldar los procesos y la información a lo largo de la cadena de suministro de alimentos (CBI, 2020).

A continuación, en la tabla 7, 8, 9 y 10 se reflejan datos de los países importadores de productos como: quinua, chía, arándanos y jengibre, por cantidad importada en toneladas, comparando los años 2018 y 2019. Se determina el crecimiento de las importaciones en puntos porcentuales para así definir los mercados más importantes para la exportación de los superalimentos seleccionados.

\section{Tabla 7}

Principales países importadores de quinua

\begin{tabular}{lcrc}
\hline \multirow{2}{*}{\multicolumn{1}{c}{ País }} & \multicolumn{2}{c}{ Toneladas importadas } & \multirow{2}{c}{$\begin{array}{c}\text { Tasa de } \\
\text { crecimiento }(\%)\end{array}$} \\
\cline { 2 - 3 } & $\mathbf{2 0 1 8}$ & $\mathbf{2 0 1 9}$ & -6 \\
Estados Unidos & 33.677 & 31.775 & 11 \\
Francia & 8.088 & 9.008 & -5 \\
Canadá & 9.005 & 8.548 & 13 \\
Alemania & 5.852 & 6.613 & 3 \\
Países Bajos & 5.324 & 5.509 & 15 \\
Reino Unido & 4.051 & 4.663 & -1 \\
España & 3.835 & 3.797 & 23 \\
Bélgica & 2.739 & 3.373 & 24 \\
Australia & 2.711 & 3.349 & -1 \\
Chile & 3.287 & 3.264 & \\
\hline
\end{tabular}

Nota. Elaboración propia, adaptado de ITC (2020b), Trade Map.

De acuerdo a lo expuesto en la tabla 7, se evidencia que el mayor importador de quinua a nivel mundial es Estados Unidos, pese a que en el año 2019 registró una tasa negativa de 
crecimiento del -6\%. Se destaca el crecimiento de la demanda por encima del 11\% en países como Francia, Alemania y Reino Unido.

\section{Tabla 8}

Principales países importadores de chía

\begin{tabular}{|c|c|c|c|}
\hline \multirow{2}{*}{ País } & \multicolumn{2}{|c|}{ Toneladas importadas } & \multirow{2}{*}{$\begin{array}{c}\text { Tasa de } \\
\text { crecimiento }(\%)\end{array}$} \\
\hline & 2018 & 2019 & \\
\hline Estados Unidos & 54.685 & 45.365 & -17 \\
\hline Francia & 8.448 & 8.779 & 4 \\
\hline Canadá & 7.210 & 7.3 .92 & 3 \\
\hline Alemania & 41.401 & 40.093 & -3 \\
\hline Países Bajos & 27.887 & 37.180 & 33 \\
\hline Reino Unido & 16.675 & 14.339 & -14 \\
\hline Bélgica & 7.199 & 6.622 & -8 \\
\hline Australia & 6.078 & 5.482 & -10 \\
\hline Chile & 2.024 & 2.364 & 17 \\
\hline China & 12.481 & 12.689 & 2 \\
\hline
\end{tabular}

Nota. Elaboración propia, adaptado de ITC (2020c), Trade Map.

En la tabla 8 se presentan los países importadores de semillas de chía. En este grupo de países resalta el volumen de compras de Estados Unidos, posicionado como el mayor importador de chía, aunque refleja una desaceleración del -17\% en el período 2018-2019. En el caso de Alemania se sitúa como segundo mayor importador, registrando un alto valor de toneladas importadas, aunque de manera similar muestra un decrecimiento en las compras en comparación al período de análisis. Cabe destacar que Países Bajos reflejan un crecimiento del 33\% en sus compras. Estos países representan un mercado de alto potencial para nuevos exportadores, considerando que su población cuenta con un alto poder adquisitivo.

\section{Tabla 9}

Principales países importadores de arándanos

\begin{tabular}{|c|c|c|c|}
\hline \multirow{2}{*}{ País } & \multicolumn{2}{|c|}{ Toneladas importadas } & \multirow{2}{*}{$\begin{array}{c}\text { Tasa de } \\
\text { crecimiento }(\%)\end{array}$} \\
\hline & 2018 & 2019 & \\
\hline Estados Unidos & 252.127 & 252.332 & 0 \\
\hline Francia & 10.108 & 12.888 & 28 \\
\hline Canadá & 42.763 & 62.665 & 47 \\
\hline Alemania & 42.555 & 61.035 & 43 \\
\hline Países Bajos & 46.037 & 78.136 & 70 \\
\hline Reino Unido & 48.366 & 56.528 & 17 \\
\hline España & 17.496 & 21.046 & 20 \\
\hline Bélgica & 6.180 & 7.313 & 18 \\
\hline Australia & 1.134 & 1.537 & 36 \\
\hline China & 15.372 & 22.045 & 43 \\
\hline
\end{tabular}

Nota. Elaboración propia, adaptado de ITC (2020d), Trade Map. 
Según lo expuesto en la tabla 9, se puede observar que el mayor importador de arándanos es Estados Unidos, manteniendo su volumen de compras en el período 2018-2019.

Entre la lista de países descritos en la tabla, se puede apreciar que su consumo de importación ha tenido un crecimiento generalizado. De esta forma, se registran tasas de crecimiento del (70\%) en Países Bajos, (47\%) en Canadá, (43\%) Alemania y China, (20\%) España y (17\%) Reino Unido, siendo los principales destinos de las exportaciones de arándanos a nivel mundial.

Este comportamiento de los mercados se sustenta en el creciente interés de la población por una fruta de alto contenido nutricional y que generen mejores condiciones para la salud.

De acuerdo al CBI (2019) el interés de los consumidores se está enfocando en los arándanos de producción orgánica y certificados, así los minoristas como Carrefour, presente en la mayoría de países europeos, diversifica su oferta en variedades, calidades y marcas.

\section{Tabla 10}

Principales países importadores de jengibre

\begin{tabular}{lccc}
\hline \multirow{2}{*}{ País } & \multicolumn{2}{c}{ Toneladas importadas } & $\begin{array}{c}\text { Tasa de } \\
\text { Crecimiento (\%) }\end{array}$ \\
\cline { 2 - 3 } Estados Unidos & $\mathbf{2 0 1 8}$ & $\mathbf{2 0 1 9}$ & 1 \\
Francia & 84.714 & 85.914 & 23 \\
Canadá & 7.035 & 8.647 & 5 \\
Alemania & 16.772 & 17.534 & 12 \\
Países Bajos & 17.862 & 20.090 & 4 \\
Reino Unido & 73.339 & 76.334 & -3 \\
España & 22.921 & 22.122 & 11 \\
Pakistán & 7.428 & 8.221 & -4 \\
Japón & 92.799 & 89.249 & -1 \\
Malasia & 59.127 & 58.499 & 48 \\
\hline
\end{tabular}

Nota. Elaboración propia, adaptado de ITC (2020e), Trade Map.

La tabla 10 presenta a los principales importadores de jengibre. Entre los países que registran un aumento en sus volúmenes de importación se destacan: Malasia (48\%), Francia (23\%), Alemania (12\%), España (11\%), destinos de exportación que representan un alto potencial de mercado.

El principal mercado importador de jengibre es Estados Unidos, que lidera la lista de los 10 países de mayor importación en el período 2018-2019, se trata de un mercado atractivo y con menores obstáculos para el ingreso de productos del sector alimenticio de América Latina.

Los hallazgos, sujetos a los superalimentos seleccionados para el análisis, revelan que existen países con una demanda creciente, en este sentido, representan mercados de oportunidad para el sector exportador de alimentos. En coherencia con lo expuesto por Tacer (2019) los 
mercados con demanda de superalimentos son distantes al origen de estos productos. Lo que guarda relación con la propuesta de Magrach y Sanz (2020), considerando la creciente demanda a nivel mundial, principalmente en los países desarrollados y del continente europeo.

\section{Conclusiones}

Este trabajo se ha desarrollado bajo la premisa de abordar el dinamismo del mercado, que actualmente se refleja en el cambio del comportamiento de los consumidores, interesados por una alimentación más sana y el respeto al medio ambiente. En este contexto, el auge de la demanda por los superalimentos se inserta como una necesidad que las empresas exportadoras deben gestionar para mantener su participación en un entorno altamente competitivo.

A la interrogante de investigación planteada se ha dado respuesta con los resultados obtenidos, de los cuales se destaca que existen oportunidades para las empresas en función de la variedad de superalimentos que puedan configurar en su oferta exportable, reforzada con el cumplimiento de la normativa sanitaria de los países importadores, y una variedad de certificaciones que prioriza un segmento de mercado altamente exigente y bien informado. La aplicación de certificaciones internacionales como USDA Organics, The Rainforest Alliance, Fairtrade, Logos Veganos, entre otras; puede ser aprovechada por las empresas como mecanismo de acceso a más mercados, ya que aseguran la inocuidad de los alimentos en margen de la responsabilidad social, y normas ambientales, lo que permitiría generar un incremento en sus volúmenes de exportación.

En consonancia con el objetivo que ha guiado la investigación, específicamente, analizar la tendencia de mercado enfocada en el consumo de superalimentos, se han identificado países que representan oportunidades para las empresas exportadoras de alimentos: Estados Unidos, Alemania, Francia, España, Países Bajos, entre otro.

La revisión y análisis de las estadísticas de importación de cuatro productos, ha permitido verificar que existen mercados potenciales en varios países, principalmente del continente europeo, lo que resulta pertinente para que las empresas latinoamericanas incursionen con la oferta de sus superalimentos.

Finalmente, de acuerdo al enfoque cualitativo adoptado en esta investigación, a partir del análisis descriptivo basado en fuentes secundarias, este trabajo contribuye a enfatizar la importancia para el sector empresarial de analizar las variables sociodemográficas, así como la percepción y comportamiento del consumidor para definir el segmento o nicho de mercado destinado a la exportación de los denominados superalimentos.

Se reconoce que el enfoque cualitativo del artículo no permite generalizar los hallazgos, no obstante, se puede considerar para futuras investigaciones realizar un estudio cuantitativo que permita incorporar la percepción de los empresarios respecto a los retos que representan los cambios de comportamiento de los consumidores mundiales, y las variables que considerarían para establecer estrategias que les permita afrontar las nuevas tendencias de los mercados potenciales. 


\section{Referencias Bibliográficas}

Algara Suarez, P., Gallegos Martínez, J., y Reyes Hernández, J. (2016). El amaranto y sus efectos terapéuticos. Tlatemoani: Revista Académica de Investigación, 7(21), 55-73. https://dialnet.unirioja.es/servlet/articulo?codigo=7298394

Allievi, F., Vinnari, M., \& Luukkanen, J. (2015). Meat consumption and production - analysis of efficiency, sufficiency and consistency of global trends. Journal of Cleaner Production, 92, 142-151. https://doi.org/10.1016/j.jclepro.2014.12.075

Ancos, B., Fernández-Jalao, I., y Sánchez-Moreno, C. (2016). Compuestos funcionales en productos de IV Y V gama. Revista Iberoamericana de Tecnología Postcosecha, 17(2), 130-148. http://www.redalyc.org/articulo.oa?id=81349041002

Ayala-Garay, A. V., Espitia-Rangel, E., Altamirano-Cárdenas, J. R., Ireta-Paredes, A., GonzálezMolina, L., Muñiz-Reyes, E., \& Almaguer-Vargas, G. (2020). Factors favoring amaranth consumption in Mexico City: Case study Xochimilco. Textual (75), 75-99. http://dx.doi.org/10.5154/r.textual.2018.75.03

Bai, X., Jung, G., \& Kim, H. (2019). A Study on the Purchase Intention of Ginseng Products based on the theory of Perceived Value and TPB. Journal of the Korea Convergence Society, 10, 35-42. https://doi.org/10.15207/JKCS.2019.10.12.035

Bañares Vilella, S. (2009). Las declaraciones alimentarias en la Unión Europea: una somera aproximación jurídica. Actividad Dietética, 13(1), 31-34. https://doi.org/10.1016/S11380322(09)71396-3

Birch, D., Skallerud, K., \& Nicholas A., P. (2018). "Who are the future seaweed consumers in a Western society? Insights from Australia". British Food Journal, 121(2), 603-615. https://doi.org/10.1108/BFJ-03-2018-0189

Boratyńska, K., \& Huseynov, R. (2017). An innovative approach to food security policy in developing countries. Journal of Innovation \& Knowledge, 2(1), 39-44. https://doi.org/10.1016/j.jik.2016.01.007

Bracale, R., \& Vaccaro, C. M. (2020). Changes in food choice following restrictive measures due to Covid-19. Nutrition, Metabolism and Cardiovascular Diseases, 30(9), 1423-1426. https://doi.org/10.1016/j.numecd.2020.05.027

Brečić, R., Mesić, Z., \& Cerjak, M. (2017). Importance of intrinsic and extrinsic quality food characteristics by different consumer segments. British Food Journal, 119(4), 845-862. https://doi.org/10.1108/BFJ-06-2016-0284

Buse Thorne, E., Chong, M., y Vílchez Túpac, L. (2017). Biocomercio, comercio justo y asociatividad modelo para los superfoods peruanos. Industrial Data, 20(2), 71-77. http://www.redalyc.org/articulo.oa?id=81653909010

Camarena-Gómez, B. O., Romero-Valenzuela, D. M., y Camarena-Gómez, D. M. (2020). Alimentos orgánicos en Hermosillo, Sonora: disposición a pagar y preferencias del consumidor. Estudios Sociales, 30(55), 2-30. http://dx.doi.org/10.24836/es.v30i55.877

CBI. (2019, august 28). Which trends offer opportunities or pose threats on the European fresh fruit and vegetables market? Netherlands Ministry of Foreign Affairs: https://www.cbi.eu/node/971/pdf

CBI. (2020, february 19). Entering the European market for blueberries. Netherlands Ministry of Foreign Affairs: https://www.cbi.eu/node/1201/pdf 
Comisión Europea. (s.f.). Declaraciones sobre nutrición y salud. https://ec.europa.eu/food/safety/labelling_nutrition/claims_en

Delicato, C., Salvatore, F. P., \& Contò, F. (2019). Consumers' understanding of healthy foods: The evidence on superfoods in Belgium [Conference]. Eighth AIEAA Conference: Diet transition and its implications on health and the environment, (págs. 1-13). Pistoia, Italy. https://doi.org/10.22004/ag.econ.300907

Ekiert, K., \& Dochniak, M. (2015). Superfoods-idealne uzupełnienie diety czy zbędny dodatek. Pielegniarstwo i Zdrowie Publiczne, 5(4), 401-408. https://doi.org/10.17219/pzp/60915

European Food Information Council. (2012, december 12). What Are Superfoods and Are They Really Super?. EUFIC: https://www.eufic.org/en/healthy-living/article/the-sciencebehind-superfoods-are-they-really-super

FAO. (2017). The future of food and agriculture - Trends and challenges. http://www.fao.org/3/ai6583e.pdf

Godfray H., C. J., \& Garnett, T. (2014). Food security and sustainable intensification. Philosophical transactions of the Royal Society B: biological sciences, 369(1639), 20120273. http://dx.doi.org/10.1098/rstb.2012.0273

Godfray H., C. J., Aveyard, P., Garnett, T., Hall, J. W., Key, T. J., Lorimer, J., . . Jebb, S. A. (2018). Meat consumption, health, and the environment. Science, 361(6399), eaam5324. http://dx.doi.org/10.1126/science.aam5324

Graeff Hönninger, S., \& Khajehei, F. (2019). The Demand for Superfoods: Consumers' Desire, Production Viability and Bio-intelligent. En C. Piatti, S. Graeff Hönninger, \& F. Khajeh (Eds.). Food Tech Transitions (págs. 81-94). Springer. https://doi.org/10.1007/978-3-03021059-5

Gutiérrez Pérez, C., Tuñón Pablos, E., Limón Aguirre, F., Morales, H., y Nigh Nielsen, R. (2012). Representaciones sociales de los alimentos orgánicos entre consumidores de Chiapas. $\begin{array}{llll}\text { Estudios Sociales } & \text { (Hermosillo, } & \text { Son.), }\end{array}$ http://www.scielo.org.mx/pdf/estsoc/v20n39/v20n39a4.pdf

International Trade Center (ITC). (2020a). List of exporters for the selected product. https://www.trademap.org/Country_SelProduct_TS.aspx?nvpm=3\%7c\%7c\%7c\%7c\%7c1 $00850 \% 7 \mathrm{c} \% 7 \mathrm{c} \% 7 \mathrm{c} 6 \% 7 \mathrm{c} 1 \% 7 \mathrm{c} 1 \% 7 \mathrm{c} 2 \% 7 \mathrm{c} 2 \% 7 \mathrm{c} 1 \% 7 \mathrm{c} 2 \% 7 \mathrm{c} 2 \% 7 \mathrm{c} 1 \% 7 \mathrm{c} 1$

International Trade Center (ITC). (2020b). List of importers for the selected product. Product: 100850 Quinua "Chenopodium quinoa". https://www.trademap.org/Country_SelProduct_TS.aspx?nvpm=1\%7c\%7c\%7c\%7c\%7c1 $00850 \% 7 \mathrm{c} \% 7 \mathrm{c} \% 7 \mathrm{c} 6 \% 7 \mathrm{c} 1 \% 7 \mathrm{c} 1 \% 7 \mathrm{c} 1 \% 7 \mathrm{c} 2 \% 7 \mathrm{c} 1 \% 7 \mathrm{c} 2 \% 7 \mathrm{c} 2 \% 7 \mathrm{c} 1 \% 7 \mathrm{c} 1$

International Trade Center (ITC). (2020c). List of importers for the selected product. Product: 120799 Semillas y frutos oleaginosos, incluso quebrantados. https://www.trademap.org/Country_SelProduct_TS.aspx?nvpm=1\%7c\%7c\%7c\%7c\%7c1 $20799 \% 7 \mathrm{c} \% 7 \mathrm{c} \% 7 \mathrm{c} 6 \% 7 \mathrm{c} 1 \% 7 \mathrm{c} 1 \% 7 \mathrm{c} 1 \% 7 \mathrm{c} 2 \% 7 \mathrm{c} 1 \% 7 \mathrm{c} 2 \% 7 \mathrm{c} 2 \% 7 \mathrm{c} 1 \% 7 \mathrm{c} 1$

International Trade Center (ITC). (2020d). List of importers for the selected product. Product: 081040 Arándanos rojos, mirtilos y demás frutos del género "Vaccinium", frescos. https://www.trademap.org/Country_SelProduct_TS.aspx?nvpm=1\%7c\%7c\%7c\%7c\%7c0 $81040 \% 7 \mathrm{c} \% 7 \mathrm{c} \% 7 \mathrm{c} 6 \% 7 \mathrm{c} 1 \% 7 \mathrm{c} 1 \% 7 \mathrm{c} 1 \% 7 \mathrm{c} 2 \% 7 \mathrm{c} 1 \% 7 \mathrm{c} 2 \% 7 \mathrm{c} 2 \% 7 \mathrm{c} 1 \% 7 \mathrm{c} 1$

International Trade Center (ITC). (2020e). List of importers for the selected product. Product: 091011 Jengibre, sin triturar ni pulverizar. https://www.trademap.org/Country_SelProduct_TS.aspx?nvpm=1\%7c\%7c\%7c\%7c\%7c0 $91011 \% 7 \mathrm{c} \% 7 \mathrm{c} \% 7 \mathrm{c} 6 \% 7 \mathrm{c} 1 \% 7 \mathrm{c} 1 \% 7 \mathrm{c} 1 \% 7 \mathrm{c} 2 \% 7 \mathrm{c} 1 \% 7 \mathrm{c} 2 \% 7 \mathrm{c} 2 \% 7 \mathrm{c} 1 \% 7 \mathrm{c} 1$ 
Karelakis, C., Zevgitis, P., Galanopoulos, K., \& Mattas, K. (2019). Consumer Trends and Attitudes to Functional Foods. Journal of International Food \& Agribusiness Marketing, 32, 266294. https://w10.1080/08974438.2019.1599760

Kulczyński, B., \& Gramza-Michałowska, A. (2016). Goji berry (Lycium barbarum): composition and health effects-a review. Polish Journal of Food and Nutrition Sciences, 66(2), 67-76. https://doi.org/10.1515/pjfns-2015-0040

Küster-BoludaaI, I., \& Vidal-Capilla, I. (2017). Consumer attitudes in the election of functional foods. Spanish Journal of Marketing - ESIC, 21(1), 65-79. https://doi.org/10.1016/j.sjme.2017.05.002

Lau, T. C., Chan, M. W., \& Tan, H. P. (2013). Functional food: a growing trend among the health conscious. Asian Social Science, 9(1), 198. http://dx.doi.org/10.5539/ass.v9n1p198

Loyer, J. (2016). Superfoods. En D. M. Kaplan, \& P. B. Thompson (Eds.), Encyclopedia of food and agricultural ethics (2nd ed., págs. 1-7). Springer. https://doi.org/10.1007 / 978-94-0076167-4_574-1

Loyer, J. (2017). The Cranberry as Food, Health Food, and Superfood: Challenging or maintaining hegemonic nutrition? Graduate Journal of Food Studies, 4(2), 33-49. https://gradfoodstudies.files.wordpress.com/2017/11/loyer-the-cranberry-as-food.pdf

Loyer, J., \& Knight, C. (2018). Selling the "Inca superfood": nutritional primitivism in superfoods books and maca marketing. Food, Culture \& Society, 21(4), 449-467. https://doi.org/10.1080/15528014.2018.1480645

Magrach, A., \& Sanz, M. (2020). Environmental and social consequences of the increase in the demand for 'superfoods' world-wide. People and Nature, 2(2), 267-278. https://doi.org/10.1002/pan3.10085

Marques, A. C., Fuinhas, J. A., \& Pais, D. F. (2018). Economic growth, sustainable development and food consumption: Evidence across different income groups of countries. Journal of Cleaner Production, 196, 245-258. https://doi.org/10.1016/j.jclepro.2018.06.011

McDonell, E. (2015). Miracle foods: Quinoa, curative metaphors, and the depoliticization of global hunger politics. Gastronomica, 15(4), 70-85. https://doi.org/10.1525/gfc.2015.15.4.70

Menezes, E., Deliza, R., Chan, H., \& Guinard, J. X. (2011). Preferences and attitudes towards açaíbased products among North American consumers. Food Research International, 44(7), 1997-2008. https://doi.org/10.1016/j.foodres.2011.02.048

Meyerding, S. G., Kürzdörfer, A., \& Gassler, B. (2018). Consumer Preferences for Superfood Ingredients - the Case of Bread in Germany. Sustainability, 10(12), 4667-4687. https://doi.org/10.3390/su10124667

Mora-Córdova, D. E., Lituma-Loja, A. A., y González-Illescas, M. L. (2020). Las certificaciones como estrategia para la competitividad de las empresas exportadoras. INNOVA Research Journal, 5(2), 113-132. https://doi.org/10.33890/innova.v5.n2.2020.1274

Nielsen, K. E. (2016). Health beneficial consumer products - status and trends. En S. Osborn, \& W. Morley (Eds.). Developing Food Products for Consumers with Specific Dietary Needs (págs. 15-42). Woodhead Publishing. https://doi.org/10.1016/B978-0-08-100329-9.000025

Organización de las Naciones Unidas para la Alimentación y la Agricultura (FAO). (2020). Seguridad Alimentaria bajo la Pandemia de COVID-19. http://www.fao.org/fileadmin/user_upload/rlc/docs/covid19/Boletin-FAO-CELAC.pdf 
Palmieria, N., \& Forleo, M. B. (2020). The potential of edible seaweed within the western diet. A segmentation of Italian consumers. International Journal of Gastronomy and Food Science, 20, 100202. https://doi.org/10.1016/j.ijgfs.2020.100202

Piatti, C., Graeff-Hönninger, S., \& Khajehei, F. (Eds.). (2019). Food Tech Transitions. Springer. https://doi.org/10.1007/978-3-030-21059-5

Procolombia. (09 de julio de 2018). Guía práctica para conocer las certificaciones que se requieren para exportación de agroalimentos. http://www.procolombia.co/publicaciones/guia-practica-para-conocer-las-certificacionesque-se-requieren-para-exportacion-de-agroalimentos

Proecuador. (25 de junio de 2013). Guía de certificaciones internacionales. https://issuu.com/proecuador/docs/guiacertificacionesh

Regulation (EC) $\mathrm{N}^{\circ}$ 1924/2006 of European Parlamient and of the Council. (2006, december 30). Official Journal L. 404. On nutrition and health claims made on foods. European Parliament and the Council of the European Union. https://eur-lex.europa.eu/legalcontent/EN/TXT/PDF/?uri=CELEX:02006R1924-20141213

Rizo-Mustelier, M., Vuelta-Lorenzo, D. R., y Lorenzo-García, A. M. (abril-junio de 2017). Agricultura, desarrollo sostenible, medioambiente, saber campesino y universidad. Ciencia en su PC(2), 106-120. http://www.redalyc.org/articulo.oa?id=181351615008

Rojas-Rivas, E., Espinoza-Ortegab, A., Thomé-Ortíz, H., Moctezuma-Pérez, S., \& Cuffia, F. (2019). Understanding consumers' perception and consumption motives towards amaranth in Mexico using the Pierre Bourdieu's theoretical concept of Habitus. Appetite, 139, 180188. https://doi.org/10.1016/j.appet.2019.04.021

Salgado Beltrán, L. (2019). Segmentación de los consumidores de alimentos orgánicos según sus actitudes, valores y creencias ambientales. Contaduría y administración, 64(2), 1-22. http://dx.doi.org/10.22201/fca.24488410e.2018.1491

Sheng, J., Shen, L., Qiao, Y., Yu, M., \& Fan, B. (2009). Market trends and accreditation systems for organic food in China. Trends in Food Science \& Technology, 20(9), 396-401. https://doi.org/10.1016/j.tifs.2009.01.053

SI, L. W. (2020). Trending foods and beverages. En M. Gibson (Ed.). Food and Society. (págs. 305-321.). Academic Press. https://doi.org/10.1016/B978-0-12-811808-5.00016-7

Soni, R. A., Sudhakar, K., \& Rana, R. S. (2017). Spirulina-From growth to nutritional product: A review. Trends in food science \& technology, 69, 157-171. https://doi.org/10.1016/j.tifs.2017.09.010

Sosa, A., Ruiz, G., Rana, J., Gordillo, G., West, H., Sharma, M., . . . Robles de la Torre, R. R. (2016). Chia Crop (Salvia hispanica L.): its History and Importance as a Source of Polyunsatu-rated Fatty Acids Omega-3 Around the World: a Review. Journal of Crop Research and Fertilizers, 1, 1-9. https://doi.org/10.17303/JCRF.2016.104

Sulistyawati, I., Dekker, M., Verkerk, R., \& Steenbekkers, B. (2020). Consumer preference for dried mango attributes: A conjoint study among Dutch, Chinese, and Indonesian consumers. Journal of food science. https://doi.org/10.1111/1750-3841.15439

Tacer Caba, Z. (2019). The concept of superfoods in diet. En C. Galanakis (Ed.), The Role of Alternative and Innovative Food Ingredients and Products in Consumer Wellness (pág. 73). Academic Press. https://doi.org/10.1016/B978-0-12-816453-2.00003-6

Tichy, H. V., Bruhs, A., \& Palisch, A. (2020). Development of real-time PCR systems for the detection of so-called "superfoods" Chia and Quinoa in commercial food products. Journal of Agricultural and Food Chemistry. https://doi.org/10.1021/acs.jafc.0c02441 
Tilman, D., \& Clark, M. (2014). Global diets link environmental sustainability and human health. Nature, 515(7528), 518-522. https://doi.org/10.1038/nature13959

Van den Driessche, J. J., Plat, J., \& Mensink, R. P. (2018). Effects of superfoods on risk factors of metabolic syndrome: a systematic review of human intervention trials. Food \& function, 9(4), 1944-1966. https://doi.org/10.1039/C7FO01792H

Varzakas, T., Kandylis, P., Dimitrellou, D., Salamoura, C., Zakynthinos, G., \& Proestos, C. (2018). Innovative and fortified food: Probiotics, prebiotics, GMOs, and superfood. In Preparation and Processing of Religious and Cultural Foods, 67-129. https://doi.org/10.1016/B978-008-101892-7.00006-7

Vera, J. A., y Cañón, J. E. (2018). El valor agregado de un sistema de gestión ambiental más allá de la certificación. Revista de la Facultad de Ciencias Básicas, 16(1), 86-91. https://doi.org/10.24054/01204211.v1.n1.2018.2929

Vos, R., \& Bellù, L. G. (2019). Global trends and challenges to food and agriculture into the 21 st century. En FAO, C. Campanhola, \& S. Pandey (Eds.), Sustainable food and agriculture (págs. 11-30). Academic Press. https://doi.org/10.1016/B978-0-12-812134-4.00002-9

Wolfe, D. (2009). Wolfe, D. (2009). Superfoods: the food and medicine of the future. North Atlantic Books.

Wyman, O. (2019). Surprises in store. Fruit logistical trend report 2019. Fruitnet Media International. https://www.fruitlogistica.com/media/fl/fl_images/fl_images_press/6/pressemitteilungen_ 3/Fruit_Logistica_Trend_Report_2019.pdf

Xin, L., \& Seo, S. S. (2019). The role of consumer ethnocentrism, country image, and subjective knowledge in predicting intention to purchase imported functional foods. British Food Journal, 122(2), 448-464. https://doi.org/10.1108/BFJ-05-2019-0326 